\title{
Permanence in a Discrete Mutualism Model with Infinite Deviating Arguments and Feedback Controls
}

\author{
Changjin $X u^{1}$ and Yusen $W u^{2}$ \\ ${ }^{1}$ Guizhou Key Laboratory of Economics System Simulation, School of Mathematics and Statistics, \\ Guizhou University of Finance and Economics, Guiyang 550004, China \\ ${ }^{2}$ School of Mathematics and Statistics, Henan University of Science and Technology, Luoyang 471023, China
}

Correspondence should be addressed to Changjin Xu; xcj403@126.com

Received 22 March 2013; Accepted 4 September 2013

Academic Editor: Zhan Zhou

Copyright (C) 2013 C. Xu and Y. Wu. This is an open access article distributed under the Creative Commons Attribution License, which permits unrestricted use, distribution, and reproduction in any medium, provided the original work is properly cited.

We propose and deal with a discrete mutualism model with infinite deviating arguments and feedback controls. Sufficient conditions which guarantee the permanence of the system are obtained by using the difference inequality theory. The paper ends with brief conclusions.

\section{Introduction}

It is well known that the long-term coexistence of species in mathematical ecology is an important and ubiquitous problem. Several mathematical concepts of coexistence of species are developed to deal with this aspect. Permanence is one important topic in these concepts. In recent years, permanence has received great attention and has been investigated in a number of notable studies. For example, Fan and $\mathrm{Li}$ [1] analyzed permanence of a delayed ratio-dependent predator-prey model with Holling type functional response. Mukherjee [2] addressed the permanence and global attractivity for facultative mutualism system with delay. Zhao and Jiang [3] focused on the permanence and extinction for nonautonomous Lotka-Volterra system. Chen [4] made a theoretical discussion on the permanence and global attractivity of Lotka-Volterra competition system with feedback control. Teng et al. [5] established the permanence criteria for a delayed discrete nonautonomous-species Kolmogorov systems. For more research on the permanence behavior of predator-prey models, one can see [6-19].

In 2007, Chen and You [20] investigated the permanence of the following two species integrodifferential model of mutualism:

$$
\frac{d N_{1}(t)}{d t}=r_{1}(t) N_{1}(t)
$$

$$
\begin{gathered}
\times\left[\frac{K_{1}(t)+\alpha_{1}(t) \int_{0}^{\infty} J_{2}(s) N_{2}(t-s) d s}{1+\int_{0}^{\infty} J_{2}(s) N_{2}(t-s) d s}\right. \\
\left.-N_{1}\left(t-\sigma_{1}(t)\right)\right], \\
\frac{d N_{2}(t)}{d t}=r_{2}(t) N_{2}(t) \\
\times\left[\begin{array}{c}
\frac{K_{2}(t)+\alpha_{2}(t) \int_{0}^{\infty} J_{1}(s) N_{1}(t-s) d s}{1+\int_{0}^{\infty} J_{1}(s) N_{1}(t-s) d s} \\
-N_{2}\left(t-\sigma_{2}(t)\right)
\end{array}\right],
\end{gathered}
$$

where $r_{i}, K_{i}, \alpha_{i}$, and $\sigma_{i}(i=1,2)$ are continuous functions bounded above and below by positive constants. $\alpha_{i}>K_{i}, i=$ 1,$2 ; J_{i} \in C([0,+\infty),[0,+\infty))$ and $\int_{0}^{\infty} J_{i}(s) d s=1, i=$ 1,2 . Using the differential inequality theory, they obtained a set of sufficient conditions to ensure the permanence of system (1). For more background and biological adjustments of system (1), one could refer to [20-24] and the references cited therein. 
Many authors [24-33] have argued that discrete time models governed by difference equations are more appropriate to describe the dynamics relationship among populations than continuous ones when the populations have nonoverlapping generations. Moreover, discrete time models can also provide efficient models of continuous ones for numerical simulations. Motivated by the above viewpoint, Li and Yang [34] considered the permanence of the following discrete model of mutualism with infinite deviating arguments:

$$
\begin{aligned}
& x_{1}(n+1) \\
& =x_{1}(n) \exp \left\{r _ { 1 } ( n ) \left[\frac{K_{1}(n)+\alpha_{1}(n) \sum_{s=0}^{\infty} J_{2}(s) x_{2}(n-s)}{1+\sum_{s=0}^{\infty} J_{2}(s) x_{2}(n-s)}\right.\right. \\
& \left.\left.-x_{1}\left(n-\sigma_{1}(n)\right)\right]\right\}, \\
& \left.x_{2}(n+1)\right] \\
& =x_{2}(n) \exp \left\{r _ { 2 } ( n ) \left[\frac{K_{2}(n)+\alpha_{2}(n) \sum_{s=0}^{\infty} J_{1}(s) x_{1}(n-s)}{1+\sum_{s=0}^{\infty} J_{1}(s) x_{1}(n-s)}\right.\right. \\
& \left.\left.-x_{2}\left(n-\sigma_{2}(n)\right)\right]\right\},
\end{aligned}
$$

where $x_{i}(n)(i=1,2)$ is the density of mutualism species $i$ at the generation, $\left\{r_{i}(n)\right\},\left\{K_{i}(n)\right\},\left\{\alpha_{i}(n)\right\},\left\{\sigma_{i}(n)\right\}(i=1,2)$, $J_{1}(n)$, and $J_{2}(n)$ are bounded nonnegative sequences. Applying the comparison theorem of difference equation and some lemmas, they derived some sufficient conditions which guarantee the permanence of system (2).

It is well known that ecosystems in the real world are continuously distributed by unpredictable forces which can result in changes in the biological parameters such as survival rates [35]. Of practical interest in ecology is the question of whether or not an ecosystem can withstand those unpredictable disturbances which persist for a finite period of time. In the language of control variables, we call the disturbance functions as control variables. To the authors' knowledge, it is the first time to deal with system (2) with feedback control.

The main objective of this paper is to investigate the following discrete mutualism model with infinite deviating arguments and feedback controls:

$$
\begin{aligned}
& x_{1}(n+1) \\
& =x_{1}(n) \exp \left\{r_{1}(n)\right. \\
& \times\left[\frac{K_{1}(n)+\alpha_{1}(n) \sum_{s=0}^{\infty} J_{2}(s) x_{2}(n-s)}{1+\sum_{s=0}^{\infty} J_{2}(s) x_{2}(n-s)}\right. \\
& \left.\left.\quad-x_{1}\left(n-\sigma_{1}(n)\right)-\beta_{1}(n) u_{1}(n)\right]\right\},
\end{aligned}
$$

$$
\begin{aligned}
& x_{2}(n+1) \\
& =x_{2}(n) \exp \left\{r_{2}(n)\right. \\
& \times\left[\frac{K_{2}(n)+\alpha_{2}(n) \sum_{s=0}^{\infty} J_{1}(s) x_{1}(n-s)}{1+\sum_{s=0}^{\infty} J_{1}(s) x_{1}(n-s)}\right. \\
& \left.\left.-x_{2}\left(n-\sigma_{2}(n)\right)-\beta_{2}(n) u_{2}(n)\right]\right\}, \\
& \Delta u_{1}(n)=-\gamma_{1}(n) u_{1}(n)+\eta_{1}(n) x_{1}(n), \\
& \Delta u_{2}(n)=-\gamma_{2}(n) u_{2}(n)+\eta_{2}(n) x_{2}(n),
\end{aligned}
$$

where $x_{i}(n)(i=1,2)$ is the density of mutualism species $i$ at the generation and $u_{i}(n)(i=1,2)$ is the control variable. $r_{1}(n), r_{2}(n), K_{1}(n), K_{2}(n), \alpha_{1}(n), \alpha_{2}(n), J_{1}(n), J_{2}(n), \sigma_{1}(n)$, and $\sigma_{2}(n)$ are bounded nonnegative sequences.

Throughout this paper, we assume that

(H) $0<r_{i}^{l} \leq r_{i}^{u}, 0<K_{i}^{l} \leq K_{i}^{u}, 0<\alpha_{i}^{l} \leq \alpha_{i}^{u}, 0<\beta_{i}^{l} \leq$ $\beta_{i}^{u}, 0<\sigma_{i}^{l} \leq \sigma_{i}^{u}, \sum_{n=0}^{\infty} J_{i}(n)=1,(i=1,2)$.

Here, for any bounded sequence $\{f(n)\}, f^{u}=\sup _{n \in N}\{f(n)\}$ and $f^{l}=\inf _{n \in N}\{f(n)\}$.

Let $\sigma=\sup _{n \in Z}\left\{\sigma_{i}(n)\right\}, \widetilde{\sigma}=\inf _{n \in Z}\left\{\sigma_{i}(n)\right\}, i=1,2$. We consider (1) together with the following initial conditions:

$$
\begin{gathered}
x_{i}(\theta)=\varphi_{i}(\theta) \geq 0, \\
\theta \in N[-\tau, 0]=\{-\tau,-\tau+1, \ldots, 0\}, \quad \varphi_{i}(0)>0 .
\end{gathered}
$$

It is not difficult to see that solutions of (1) and (4) are well defined for all $n \geq 0$ and satisfy

$$
x_{i}(n)>0, \quad \text { for } n \in Z, i=1,2 .
$$

The remainder of the paper is organized as follows. In Section 2, basic definitions and lemmas are given, some sufficient conditions for the permanence of system (1) are established. Brief conclusions are presented in Section 3.

\section{Permanence}

In order to obtain the main result of this paper, we will first state the definition of permanence and several lemmas which will be useful in the proving of the main result.

Definition 1. We say that system (1) is permanence if there are positive constants $M$ and $m$ such that for each positive solution $\left(x_{1}(n), x_{2}(n), u_{1}(n), u_{2}(n)\right)$ of system (1) satisfies

$$
\begin{array}{r}
m \leq \lim _{n \rightarrow+\infty} \inf x_{i}(n) \leq \lim _{n \rightarrow+\infty} \sup x_{i}(n) \leq M, \\
(i=1,2), \\
m \leq \lim _{n \rightarrow+\infty} \inf u_{i}(n) \leq \lim _{n \rightarrow+\infty} \sup u_{i}(n) \leq M, \\
(i=1,2) .
\end{array}
$$


Let us consider the following single species discrete model:

$$
N(n+1)=N(n) \exp (a(n)-b(n) N(n)),
$$

where $\{a(n)\}$ and $\{b(n)\}$ are strictly positive sequences of real numbers defined for $n \in N=\{0,1,2, \ldots\}$ and $0<a^{l} \leq$ $a^{u}, 0<b^{l} \leq b^{u}$. Similarly to the proofs Propositions 1 and 3 in [36], we can obtain the following Lemma 2.

Lemma 2. Any solution of system (7) with initial condition $N(0)>0$ satisfies

$$
m \leq \lim _{n \rightarrow+\infty} \inf N(n) \leq \lim _{n \rightarrow+\infty} \sup N(n) \leq M,
$$

where

$$
M=\frac{1}{b^{l}} \exp \left(a^{u}-1\right), \quad m=\frac{a^{l}}{b^{u}} \exp \left(a^{l}-b^{u} M\right) .
$$

Let us consider the first order difference equation:

$$
y(n+1)=A y(n)+B, \quad n=1,2, \ldots,
$$

where $A$ and $B$ are positive constants. Following Theorem 6.2 of L. Wang and M. Q. Wang [37, page 125], we have the following Lemma 3.

Lemma 3 (see [37]). Assume that $|A|<1$, for any initial value $y(0)$, there exists a unique solution $y(n)$ of (10) which can be expressed as follows:

$$
y(n)=A^{n}\left(y(0)-y^{*}\right)+y^{*},
$$

where $y^{*}=B /(1-A)$. Thus, for any solution $\{y(n)\}$ of system (10), $\lim _{n \rightarrow+\infty} y(n)=y^{*}$.

Lemma 4 (see [37]). Let $n \in N_{n_{0}}^{+}=\left\{n_{0}, n_{0}+1, \ldots, n_{0}+l, \ldots\right\}$, $r \geq 0$. For any fixed $n, g(n, r)$ is a nondecreasing function with respect to $r$, and for $n \geq n_{0}$, the following inequalities hold:

$$
y(n+1) \leq g(n, y(n)), \quad u(n+1) \geq g(n, u(n)) .
$$

If $y\left(n_{0}\right) \leq u\left(n_{0}\right)$, then $y(n) \leq u(n)$ for all $n \geq n_{0}$.

Proposition 5. Assume that the condition (H) holds, then

$$
\lim _{n \rightarrow+\infty} \sup x_{i}(n) \leq M_{i}, \quad \lim _{n \rightarrow+\infty} \sup u_{i}(n) \leq U_{i}, \quad i=1,2,
$$

where

$$
\begin{gathered}
M_{i}=\frac{1}{r_{i}^{l}} \exp \left\{r_{i}^{u}\left(K_{i}^{u}+\alpha_{i}^{u}\right)(\sigma+1)-1\right\}, \\
U_{i}=\frac{\eta_{i}^{u} M_{i}}{\gamma_{i}^{l}}, \quad(i=1,2) .
\end{gathered}
$$

Proof. Let $\left(x_{1}(n), x_{2}(n), u_{1}(n), u_{2}(n)\right)$ be any positive solution of system (1) with the initial condition $\left(x_{1}(0), x_{2}(0)\right.$, $\left.u_{1}(0), u_{2}(0)\right)$. It follows from the first equation of system (1) that

$$
x_{1}(n+1) \leq x_{1}(n) \exp \left\{r_{1}(n) K_{1}(n)+r_{1}(n) \alpha_{1}(n)\right\} .
$$

Let $x_{1}(n)=\exp \left\{y_{1}(n)\right\}$ then (15) is equivalent to

$$
y_{1}(n+1)-y_{1}(n) \leq r_{1}(n) K_{1}(n)+r_{1}(n) \alpha_{1}(n) .
$$

Summing both sides of (16) from $n-\sigma_{1}(n)$ to $n-1$, we have

$$
\begin{aligned}
& \sum_{j=n-\sigma_{1}(n)}^{n-1}\left(y_{1}(j+1)-y_{1}(j)\right) \\
& \quad \leq \sum_{j=n-\sigma_{1}(n)}^{n-1}\left(r_{1}(j) K_{1}(j)+r_{1}(j) \alpha_{1}(j)\right) \\
& \quad \leq r_{1}^{u}\left(K_{1}^{u}+\alpha_{1}^{u}\right) \sigma
\end{aligned}
$$

which leads to

$$
y_{1}\left(n-\sigma_{1}(n)\right) \geq y_{1}(n)-r_{1}^{u}\left(K_{1}^{u}+\alpha_{1}^{u}\right) \sigma .
$$

Then

$$
x_{1}\left(n-\sigma_{1}(n)\right) \geq x_{1}(n) \exp \left\{-r_{1}^{u}\left(K_{1}^{u}+\alpha_{1}^{u}\right) \sigma\right\} .
$$

Substituting (19) into the first equation of system (1), it follows that

$$
\begin{aligned}
& x_{1}(n+1) \\
& \leq x_{1}(n) \exp \left\{r_{1}(n)\left(k_{1}(n)+\alpha_{1}(n)\right)\right. \\
& \left.\quad-r_{1}(n) \exp \left\{-r_{1}^{u}\left(K_{1}^{u}+\alpha_{1}^{u}\right) \sigma\right\} x_{1}(n)\right\} .
\end{aligned}
$$

It follows from (20) and Lemma 2 that

$$
\lim _{n \rightarrow+\infty} \sup x_{1}(n) \leq \frac{1}{r_{1}^{l}} \exp \left\{r_{1}^{u}\left(K_{1}^{u}+\alpha_{1}^{u}\right)(\sigma+1)-1\right\}:=M_{1} \text {. }
$$

For any positive constant $\varepsilon>0$, it follows (21) that there exists a $N_{1}>0$ such that for all $n>N_{1}+\sigma$

$$
x_{1}(n) \leq M_{1}+\varepsilon .
$$

From the second equation of system (1), we get

$$
x_{2}(n+1) \leq x_{2}(n) \exp \left\{r_{2}(n) K_{2}(n)+r_{2}(n) \alpha_{2}(n)\right\} .
$$

Let $x_{2}(n)=\exp \left\{y_{2}(n)\right\}$ then (23) is equivalent to

$$
y_{2}(n+1)-y_{2}(n) \leq r_{2}(n) K_{2}(n)+r_{2}(n) \alpha_{2}(n) .
$$

Summing both sides of (24) from $n-\sigma_{2}(n)$ to $n-1$, we have

$$
\begin{aligned}
& \sum_{j=n-\sigma_{2}(n)}^{n-1}\left(y_{2}(j+1)-y_{2}(j)\right) \\
& \quad \leq \sum_{j=n-\sigma_{2}(n)}^{n-1}\left(r_{2}(j) K_{2}(j)+r_{2}(j) \alpha_{2}(j)\right) \\
& \quad \leq r_{2}^{u}\left(K_{2}^{u}+\alpha_{2}^{u}\right) \sigma
\end{aligned}
$$


which leads to

$$
y_{2}\left(n-\sigma_{2}(n)\right) \geq y_{2}(n)-r_{2}^{u}\left(K_{2}^{u}+\alpha_{2}^{u}\right) \sigma .
$$

Then

$$
x_{2}\left(n-\sigma_{2}(n)\right) \geq x_{2}(n) \exp \left\{-r_{2}^{u}\left(K_{2}^{u}+\alpha_{2}^{u}\right) \sigma\right\} .
$$

Substituting (27) into the second equation of system (1), it follows that

$$
\begin{aligned}
& x_{2}(n+1) \\
& \begin{aligned}
\leq x_{2}(n) \exp \{ & r_{2}(n)\left(k_{2}(n)+\alpha_{2}(n)\right) \\
& \left.-r_{2}(n) \exp \left\{-r_{2}^{u}\left(K_{2}^{u}+\alpha_{2}^{u}\right) \sigma\right\} x_{2}(n)\right\} .
\end{aligned}
\end{aligned}
$$

It follows from (28) and Lemma 2 that

$$
\lim _{n \rightarrow+\infty} \sup x_{2}(n) \leq \frac{1}{r_{2}^{l}} \exp \left\{r_{1}^{u}\left(K_{2}^{u}+\alpha_{2}^{u}\right)(\sigma+1)-1\right\}:=M_{2} \text {. }
$$

For any positive constant $\varepsilon>0$, it follows (29) that there exists a $N_{2}>N_{1}+\sigma$ such that for all $n>N_{2}+\sigma$

$$
x_{2}(n) \leq M_{2}+\varepsilon .
$$

In view of the third and fourth equations of system (1), we can obtain

$$
\begin{aligned}
& \Delta u_{1}(n) \leq-\gamma_{1}(n) u_{1}(n)+\eta_{1}(n)\left(M_{1}+\varepsilon\right), \\
& \Delta u_{2}(n) \leq-\gamma_{2}(n) u_{2}(n)+\eta_{2}(n)\left(M_{2}+\varepsilon\right) .
\end{aligned}
$$

Then

$$
\begin{aligned}
& u_{1}(n+1) \leq\left(1-\gamma_{1}^{l}\right) u_{1}(n)+\eta_{1}^{u}\left(M_{1}+\varepsilon\right), \\
& u_{2}(n+1) \leq\left(1-\gamma_{2}^{l}\right) u_{2}(n)+\eta_{2}^{u}\left(M_{2}+\varepsilon\right) .
\end{aligned}
$$

Applying Lemmas 3 and 4, it immediately follows that

$$
\begin{aligned}
& \lim _{n \rightarrow+\infty} \sup u_{1}(n) \leq \frac{\eta_{1}^{u}\left(M_{1}+\varepsilon\right)}{\gamma_{1}^{l}}, \\
& \lim _{n \rightarrow+\infty} \sup u_{2}(n) \leq \frac{\eta_{2}^{u}\left(M_{2}+\varepsilon\right)}{\gamma_{2}^{l}} .
\end{aligned}
$$

Setting $\varepsilon \rightarrow 0$, it follows that

$$
\begin{aligned}
& \lim _{n \rightarrow+\infty} \sup u_{1}(n) \leq \frac{\eta_{1}^{u} M_{1}}{\gamma_{1}^{l}}:=U_{1}, \\
& \lim _{n \rightarrow+\infty} \sup u_{2}(n) \leq \frac{\eta_{2}^{u} M_{2}}{\gamma_{2}^{l}}:=U_{2} .
\end{aligned}
$$

This completes the proof of Proposition 5.

Theorem 6. Assume that (H) holds, then system (1) is permanent.
Proof. By applying Proposition 5, we can easily see that to end the proof of Theorem 6, it is enough to show that under the conditions of Theorem 6 ,

$$
\begin{array}{ll}
\lim _{n \rightarrow+\infty} \inf x_{2}(n) \geq m_{1}, & \lim _{n \rightarrow+\infty} \inf x_{2}(n) \geq m_{2}, \\
\lim _{n \rightarrow+\infty} \inf u_{1}(n) \geq v_{1}, & \lim _{n \rightarrow+\infty} \inf u_{2}(n) \geq v_{2} .
\end{array}
$$

In view of Proposition 5, for all $\varepsilon>0$, there exists a $N_{3}>0$, $N_{3} \in N$, for all $n>N_{3}$ :

$$
\begin{array}{ll}
x_{1}(n) \leq M_{1}+\varepsilon, & x_{2}(n) \leq M_{2}+\varepsilon, \\
u_{1}(n) \leq U_{1}+\varepsilon, & u_{2}(n) \leq U_{2}+\varepsilon .
\end{array}
$$

It follows from the first equation of systems (1) and (36) that

$$
\begin{aligned}
& x_{1}(n+1) \\
& \geq x_{1}(n) \exp \left\{r _ { 1 } ( n ) \left[\frac{K_{1}(n)}{1+\sum_{s=0}^{\infty} J_{2}(s) x_{2}(n-s)}\right.\right. \\
& \left.\left.-x_{1}\left(n-\sigma_{1}(n)\right)\right]\right\} \\
& \geq x_{1}(n) \exp \left\{\frac{r_{1}^{l} K_{1}^{l}}{1+M_{2}+\varepsilon}-\left(M_{1}+\varepsilon\right)\right\}
\end{aligned}
$$

for all $n>N_{3}+\sigma$.

Let $x_{1}(n)=\exp \left\{y_{1}(n)\right\}$; then (37) is equivalent to

$$
y_{1}(n+1)-y_{1}(n) \geq \frac{r_{1}^{l} K_{1}^{l}}{1+M_{2}+\varepsilon}-\left(M_{1}+\varepsilon\right) .
$$

Summing both sides of (38) from $n-\delta_{1}(n)$ to $n-1$ leads to

$$
\begin{aligned}
& \sum_{j=n-\delta_{1}(n)}^{n-1}\left(y_{1}(j+1)-y_{1}(j)\right) \\
& \geq \sum_{j=n-\delta_{1}(n)}^{n-1}\left[\frac{r_{1}^{l} K_{1}^{l}}{1+M_{2}+\varepsilon}-\left(M_{1}+\varepsilon\right)\right] \\
& \geq\left[\frac{r_{1}^{l} K_{1}^{l}}{1+M_{2}+\varepsilon}-\left(M_{1}+\varepsilon\right)\right] \widetilde{\sigma} .
\end{aligned}
$$

Then

$$
y_{1}\left(n-\delta_{1}(n)\right) \leq y_{1}(n)-\left[\frac{r_{1}^{l} K_{1}^{l}}{1+M_{2}+\varepsilon}-\left(M_{1}+\varepsilon\right)\right] \widetilde{\sigma} .
$$

Thus

$$
\begin{aligned}
& x_{1}\left(n-\delta_{1}(n)\right) \\
& \quad \leq x_{1}(n) \exp \left\{-\left[\frac{r_{1}^{l} K_{1}^{l}}{1+M_{2}+\varepsilon}-\left(M_{1}+\varepsilon\right)\right] \widetilde{\sigma}\right\} .
\end{aligned}
$$


Substituting (36) and (41) into the first equation of (1), we have

$$
\begin{aligned}
& x_{1}(n+1) \\
& \geq x_{1}(n) \exp \left\{r _ { 1 } ( n ) \left[\frac{K_{1}(n)}{1+\sum_{s=0}^{\infty} J_{2}(s) x_{2}(n-s)}\right.\right. \\
& \left.\left.\quad-x_{1}\left(n-\sigma_{1}(n)\right)\right]\right\} \\
& \geq x_{1}(n) \exp \left\{\frac{r_{1}^{l} K_{1}^{l}-x_{1}(n)}{1+M_{2}+\varepsilon}\right. \\
& \left.\quad \times \exp \left\{-\left[\frac{r_{1}^{l} K_{1}^{l}}{1+M_{2}+\varepsilon}-\left(M_{1}+\varepsilon\right)\right] \tilde{\sigma}\right\}\right\}
\end{aligned}
$$

for all $n>N_{3}+\sigma$.

By applying Lemmas 2 and 4, it immediately follows that

$$
\lim _{n \rightarrow+\infty} \inf x_{1}(n) \geq m_{1}^{\varepsilon}
$$

where

$$
\begin{aligned}
m_{1}^{\varepsilon}= & \frac{r_{1}^{l} K_{1}^{l}}{r_{1}^{u}\left(1+M_{1}+\varepsilon\right)} \\
& \times \exp \left\{\frac{r_{1}^{l} K_{1}^{l}}{1+M_{1}+\varepsilon}-r_{1}^{u}\right. \\
& \left.\quad \times \exp \left[-\left(\frac{r_{1}^{l} K_{1}^{l}}{1+M_{2}+\varepsilon}-\left(M_{1}+\varepsilon\right)\right) \widetilde{\sigma}\right] M_{1}\right\} .
\end{aligned}
$$

Setting $\varepsilon \rightarrow 0$ in (43), then

$$
\lim _{n \rightarrow+\infty} \inf x_{1}(n) \geq m_{1}
$$

where

$$
\begin{aligned}
m_{1}= & \frac{r_{1}^{l} K_{1}^{l}}{r_{1}^{u}\left(1+M_{1}\right)} \\
& \times \exp \left\{\frac{r_{1}^{l} K_{1}^{l}}{1+M_{1}}-r_{1}^{u}\right. \\
& \left.\quad \times \exp \left[-\left(\frac{r_{1}^{l} K_{1}^{l}}{1+M_{2}}-M_{1}\right) \widetilde{\sigma}\right] M_{1}\right\} .
\end{aligned}
$$

By the second equation of systems (1) and (36), we can obtain

$$
\begin{aligned}
x_{2}(n+1) \geq & x_{2}(n) \\
& \times \exp \left\{r _ { 2 } ( n ) \left[\frac{K_{2}(n)}{1+\sum_{s=0}^{\infty} J_{2}(s) x_{2}(n-s)}\right.\right. \\
& \left.\left.-x_{2}\left(n-\sigma_{2}(n)\right)\right]\right\} \\
\geq & x_{2}(n) \exp \left\{\frac{r_{2}^{l} K_{2}^{l}}{1+M_{1}+\varepsilon}-\left(M_{2}+\varepsilon\right)\right\}
\end{aligned}
$$

Let $x_{2}(n)=\exp \left\{y_{2}(n)\right\}$; then (47) is equivalent to

$$
y_{2}(n+1)-y_{2}(n) \geq \frac{r_{2}^{l} K_{2}^{l}}{1+M_{1}+\varepsilon}-\left(M_{2}+\varepsilon\right) .
$$

Summing both sides of (48) from $n-\delta_{2}(n)$ to $n-1$ leads to

$$
\begin{aligned}
& \sum_{j=n-\delta_{2}(n)}^{n-1}\left(y_{2}(n+1)-y_{2}(n)\right) \\
& \geq \sum_{j=n-\delta_{2}(n)}^{n-1}\left[\frac{r_{2}^{l} K_{2}^{l}}{1+M_{1}+\varepsilon}-\left(M_{2}+\varepsilon\right)\right] \\
& \geq\left[\frac{r_{2}^{l} K_{2}^{l}}{1+M_{1}+\varepsilon}-\left(M_{2}+\varepsilon\right)\right] \widetilde{\sigma} .
\end{aligned}
$$

Then

$$
y_{2}\left(n-\delta_{2}(n)\right) \leq y_{2}(n)-\left[\frac{r_{2}^{l} K_{2}^{l}}{1+M_{1}+\varepsilon}-\left(M_{2}+\varepsilon\right)\right] \tilde{\sigma}
$$

Thus

$$
\begin{aligned}
& x_{2}\left(n-\delta_{2}(n)\right) \\
& \quad \leq x_{2}(n) \exp \left\{-\left[\frac{r_{2}^{l} K_{2}^{l}}{1+M_{1}+\varepsilon}-\left(M_{2}+\varepsilon\right)\right] \widetilde{\sigma}\right\} .
\end{aligned}
$$

Substituting (36) and (51) into the second equation of (1), we have

$$
\begin{aligned}
& x_{2}(n+1) \\
& \begin{array}{l}
\geq x_{2}(n) \exp \left\{r _ { 2 } ( n ) \left[\frac{K_{2}(n)}{1+\sum_{s=0}^{\infty} J_{1}(s) x_{1}(n-s)}\right.\right. \\
\left.\left.\quad-x_{2}\left(n-\sigma_{2}(n)\right)\right]\right\} \\
\geq x_{2}(n) \exp \left\{\frac{r_{2}^{l} K_{2}^{l}-x_{2}(n)}{1+M_{1}+\varepsilon}\right. \\
\left.\quad \times \exp \left\{-\left[\frac{r_{2}^{l} K_{2}^{l}}{1+M_{1}+\varepsilon}-\left(M_{2}+\varepsilon\right)\right] \widetilde{\sigma}\right\}\right\}
\end{array}
\end{aligned}
$$

for all $n>N_{3}+\sigma$.

By applying Lemmas 2 and 4, it immediately follows that

$$
\lim _{n \rightarrow+\infty} \inf x_{2}(n) \geq m_{2}^{\varepsilon},
$$

where

$$
\begin{aligned}
m_{2}^{\varepsilon}= & \frac{r_{2}^{l} K_{2}^{l}}{r_{2}^{u}\left(1+M_{2}+\varepsilon\right)} \\
& \times \exp \left\{\frac{r_{2}^{l} K_{2}^{l}}{1+M_{2}+\varepsilon}-r_{2}^{u}\right. \\
& \left.\times \exp \left[-\left(\frac{r_{2}^{l} K_{2}^{l}}{1+M_{1}+\varepsilon}-\left(M_{2}+\varepsilon\right)\right) \widetilde{\sigma}\right] M_{2}\right\} .
\end{aligned}
$$

for all $n>N_{3}+\sigma$. 
Setting $\varepsilon \rightarrow 0$ in (53), then

$$
\lim _{n \rightarrow+\infty} \inf x_{2}(n) \geq m_{2}
$$

where

$$
\begin{aligned}
m_{2}= & \frac{r_{2}^{l} K_{2}^{l}}{r_{2}^{u}\left(1+M_{2}\right)} \\
& \quad \times \exp \left\{\frac{r_{2}^{l} K_{2}^{l}}{1+M_{2}}-r_{2}^{u}\right. \\
& \left.\quad \times \exp \left[-\left(\frac{r_{2}^{l} K_{2}^{l}}{1+M_{1}}-M_{2}\right) \widetilde{\sigma}\right] M_{2}\right\} .
\end{aligned}
$$

Without loss of generality, we assume that $\varepsilon<(1 / 2) \min \left\{m_{1}\right.$, $\left.m_{2}\right\}$. For any positive constant $\varepsilon$ small enough, it follows from (45) and (55) that there exists enough large $N_{4}>N_{3}+\sigma$ such that

$$
x_{1}(n) \geq m_{1}-\varepsilon, \quad x_{2}(n) \geq m_{2}-\varepsilon
$$

for any $n \geq N_{4}$.

From the third and fourth equations of systems (1) and (57), we can derive that

$$
\begin{aligned}
& \Delta u_{1}(n) \geq-\gamma_{1}(n) u_{1}(n)+\eta_{1}(n)\left(m_{1}-\varepsilon\right), \\
& \Delta u_{2}(n) \geq-\gamma_{2}(n) u_{2}(n)+\eta_{2}(n)\left(m_{2}-\varepsilon\right) .
\end{aligned}
$$

Hence

$$
\begin{aligned}
& u_{1}(n+1) \geq\left(1-\gamma_{1}^{u}\right) u_{1}(n)+\eta_{1}^{l}\left(m_{1}-\varepsilon\right), \\
& u_{2}(n+1) \geq\left(1-\gamma_{2}^{u}\right) u_{2}(n)+\eta_{2}^{l}\left(m_{2}-\varepsilon\right) .
\end{aligned}
$$

By applying Lemmas 2 and 3, it immediately follows that

$$
\begin{aligned}
& \lim _{n \rightarrow+\infty} \inf u_{1}(n) \geq \frac{\eta_{1}^{l}\left(m_{1}-\varepsilon\right)}{\gamma_{1}^{u}}, \\
& \lim _{n \rightarrow+\infty} \inf u_{2}(n) \geq \frac{\eta_{2}^{l}\left(m_{2}-\varepsilon\right)}{\gamma_{2}^{u}} .
\end{aligned}
$$

Setting $\varepsilon \rightarrow 0$ in the previous inequality leads to

$$
\begin{aligned}
& \lim _{n \rightarrow+\infty} \inf u_{1}(n) \geq \frac{\eta_{1}^{l} m_{1}}{\gamma_{1}^{u}}, \\
& \lim _{n \rightarrow+\infty} \inf u_{2}(n) \geq \frac{\eta_{2}^{l} m_{2}}{\gamma_{2}^{u}} .
\end{aligned}
$$

This completes the proof of Theorem 6 .

\section{Conclusions}

In the present paper, we have investigated the permanence of a discrete mutualism model with infinite deviating arguments and feedback controls. Sufficient conditions which ensure the permanence of the system are established. We have shown the effect of delay to the permanence of system and concluded that delay is an important factor to decide the permanence of the system.

\section{Acknowledgments}

This work is supported by thr National Natural Science Foundation of China (no. 11261010 and no. 11101126), the Soft Science and Technology Program of Guizhou Province (no. 2011LKC2030), the Natural Science and Technology Foundation of Guizhou Province (J[2012]2100), the Governor Foundation of Guizhou Province ([2012]53), and the Doctoral Foundation of Guizhou University of Finance and Economics (2010).

\section{References}

[1] Y.-H. Fan and W.-T. Li, "Permanence for a delayed discrete ratio-dependent predator-prey system with Holling type functional response," Journal of Mathematical Analysis and Applications, vol. 299, no. 2, pp. 357-374, 2004.

[2] D. Mukherjee, "Permanence and global attractivity for facultative mutualism system with delay," Mathematical Methods in the Applied Sciences, vol. 26, no. 1, pp. 1-9, 2003.

[3] J. D. Zhao and J. F. Jiang, "Average conditions for permanence and extinction in nonautonomous Lotka-Volterra system," Journal of Mathematical Analysis and Applications, vol. 299, no. 2, pp. 663-675, 2004.

[4] F. D. Chen, "The permanence and global attractivity of LotkaVolterra competition system with feedback controls," Nonlinear Analysis. Real World Applications, vol. 7, no. 1, pp. 133-143, 2006.

[5] Z. D. Teng, Y. Zhang, and S. J. Gao, "Permanence criteria for general delayed discrete nonautonomous $n$-Kolmogorov systems and its applications," Computers \& Mathematics with Applications, vol. 59, no. 2, pp. 812-828, 2010.

[6] F. D. Chen, "Permanence of a discrete $n$-species food-chain system with time delays," Applied Mathematics and Computation, vol. 185, no. 1, pp. 719-726, 2007.

[7] J. Dhar and K. S. Jatav, "Mathematical analysis of a delayed stage-structured predator-prey model with impulsive diffsion between two predators territories," Ecological Complexity, 2012.

[8] S. Q. Liu and L. S. Chen, "Necessary-sufficient conditions for permanence and extinction in Lotka-Volterra system with distributed delays," Applied Mathematics Letters, vol. 16, no. 6, pp. 911-917, 2003.

[9] X. Y. Liao, S. F. Zhou, and Y. M. Chen, "Permanence and global stability in a discrete $n$-species competition system with feedback controls," Nonlinear Analysis. Real World Applications, vol. 9, no. 4, pp. 1661-1671, 2008.

[10] H. X. Hu, Z. D. Teng, and H. J. Jiang, "On the permanence in non-autonomous Lotka-Volterra competitive system with puredelays and feedback controls," Nonlinear Analysis. Real World Applications, vol. 10, no. 3, pp. 1803-1815, 2009.

[11] Y. Muroya, "Permanence and global stability in a Lotka-Volterra predator-prey system with delays," Applied Mathematics Letters, vol. 16, no. 8, pp. 1245-1250, 2003.

[12] Z. Y. Hou, "On permanence of Lotka-Volterra systems with delays and variable intrinsic growth rates," Nonlinear Analysis. Real World Applications, vol. 14, no. 2, pp. 960-975, 2013.

[13] X. S. Xiong and Z. Q. Zhang, "Periodic solutions of a discrete two-species competitive model with stage structure," Mathematical and Computer Modelling, vol. 48, no. 3-4, pp. 333-343, 2008.

[14] R. Y. Zhang, Z. C. Wang, Y. M. Chen, and J. H. Wu, "Periodic solutions of a single species discrete population model with 
periodic harvest/stock," Computers \& Mathematics with Applications, vol. 39, no. 1-2, pp. 77-90, 2009.

[15] Y. Takeuchi, Global Dynamical Properties of Lotka-Volterra Systems, World Scientific Publishing, River Edge, NJ, USA, 1996.

[16] Y. G. Sun and S. H. Saker, "Positive periodic solutions of discrete three-level food-chain model of Holling type II," Applied Mathematics and Computation, vol. 180, no. 1, pp. 353-365, 2006.

[17] X. H. Ding and C. Lu, "Existence of positive periodic solution for ratio-dependent $n$-species difference system," Applied Mathematical Modelling, vol. 33, no. 6, pp. 2748-2756, 2009.

[18] Z. C. Li, Q. L. Zhao, and D. Ling, "Chaos in a discrete population model," Discrete Dynamics in Nature and Society, vol. 2012, Article ID 482459, 14 pages, 2012.

[19] H. Xiang, K.-M. Yan, and B.-Y. Wang, "Existence and global stability of periodic solution for delayed discrete high-order Hopfield-type neural networks," Discrete Dynamics in Nature and Society, vol. 2005, no. 3, pp. 281-297, 2005.

[20] F. D. Chen and M. S. You, "Permanence for an integrodifferential model of mutualism," Applied Mathematics and Computation, vol. 186, no. 1, pp. 30-34, 2007.

[21] F. D. Chen, "Permanence in a discrete Lotka-Volterra competition model with deviating arguments," Nonlinear Analysis. Real World Applications, vol. 9, no. 5, pp. 2150-2155, 2008.

[22] Y. K. Li and G. T. Xu, "Positive periodic solutions for an integrodifferential model of mutualism," Applied Mathematics Letters, vol. 14, no. 5, pp. 525-530, 2001.

[23] P. Yang and R. Xu, "Global asymptotic stability of periodic solution in $n$-species cooperative system with time delays," Journal of Biomathematics, vol. 13, no. 6, pp. 841-846, 1998.

[24] Y. K. Li and H. T. Zhang, "Existence of periodic solutions for a periodic mutualism model on time scales," Journal of Mathematical Analysis and Applications, vol. 343, no. 2, pp. 818825, 2008.

[25] R. P. Agarwal, Difference Equations and Inequalities. Theory, Methods, and Applications, vol. 228 of Monographs and Textbooks in Pure and Applied Mathematics, Marcel Dekker, New York, NY, USA, 2nd edition, 2000.

[26] Y. K. Li and L. H. Lu, "Positive periodic solutions of discrete $n$-species food-chain systems," Applied Mathematics and Computation, vol. 167, no. 1, pp. 324-344, 2005.

[27] X. Chen and F. D. Chen, "Stable periodic solution of a discrete periodic Lotka-Volterra competition system with a feedback control," Applied Mathematics and Computation, vol. 181, no. 2, pp. 1446-1454, 2006.

[28] Y. Muroya, "Persistence and global stability in Lotka-Volterra delay differential systems," Applied Mathematics Letters, vol. 17, no. 7, pp. 795-800, 2004.

[29] Y. Muroya, "Partial survival and extinction of species in discrete nonautonomous Lotka-Volterra systems," Tokyo Journal of Mathematics, vol. 28, no. 1, pp. 189-200, 2005.

[30] X. T. Yang, "Uniform persistence and periodic solutions for a discrete predator-prey system with delays," Journal of Mathematical Analysis and Applications, vol. 316, no. 1, pp. 161-177, 2006.

[31] F. D. Chen, "Permanence for the discrete mutualism model with time delays," Mathematical and Computer Modelling, vol. 47, no. 3-4, pp. 431-435, 2008.

[32] W. P. Zhang, D. M. Zhu, and P. Bi, "Multiple positive periodic solutions of a delayed discrete predator-prey system with type IV functional responses," Applied Mathematics Letters, vol. 20, no. 10, pp. 1031-1038, 2007.
[33] Y. M. Chen and Z. Zhou, "Stable periodic solution of a discrete periodic Lotka-Volterra competition system," Journal of Mathematical Analysis and Applications, vol. 277, no. 1, pp. 358366, 2003.

[34] X. P. Li and W. S. Yang, "Permanence of a discrete model of mutualism with infinite deviating arguments," Discrete Dynamics in Nature and Society, vol. 2010, Article ID 931798, 7 pages, 2010.

[35] X. P. Li and W. S. Yang, "Permanence of a discrete predator-prey systems with Beddington-DeAngelis functional response and feedback controls," Discrete Dynamics in Nature and Society, vol. 2008, Article ID 149267, 8 pages, 2008.

[36] F. D. Chen, "Permanence and global attractivity of a discrete multispecies Lotka-Volterra competition predator-prey systems," Applied Mathematics and Computation, vol. 182, no. 1, pp. 3-12, 2006.

[37] L. Wang and M. Q. Wang, Ordinary Difference Equation, Xinjiang University Press, 1991. 


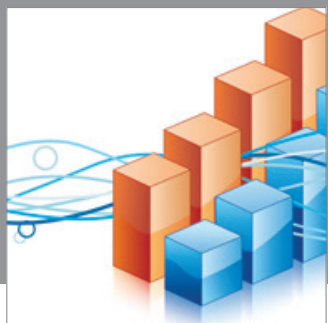

Advances in

Operations Research

mansans

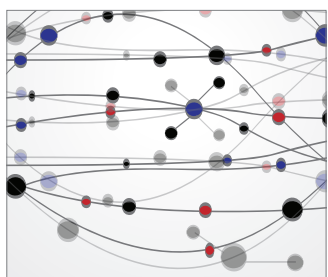

The Scientific World Journal
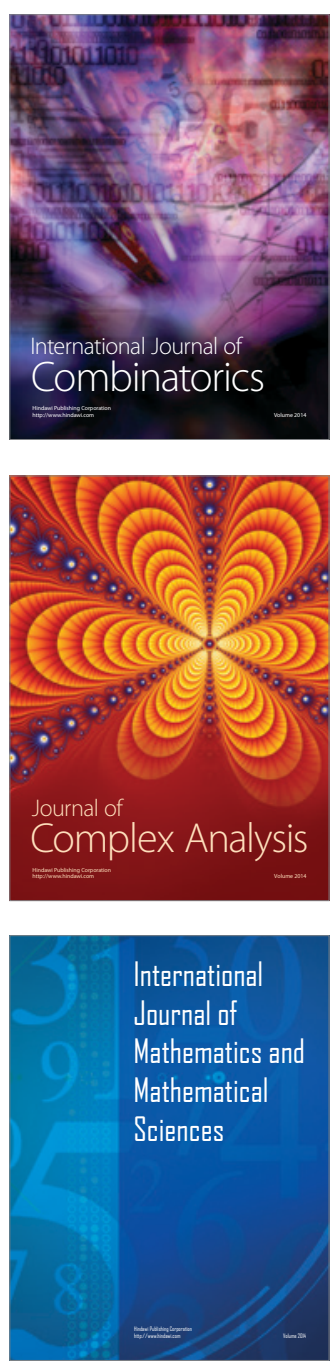
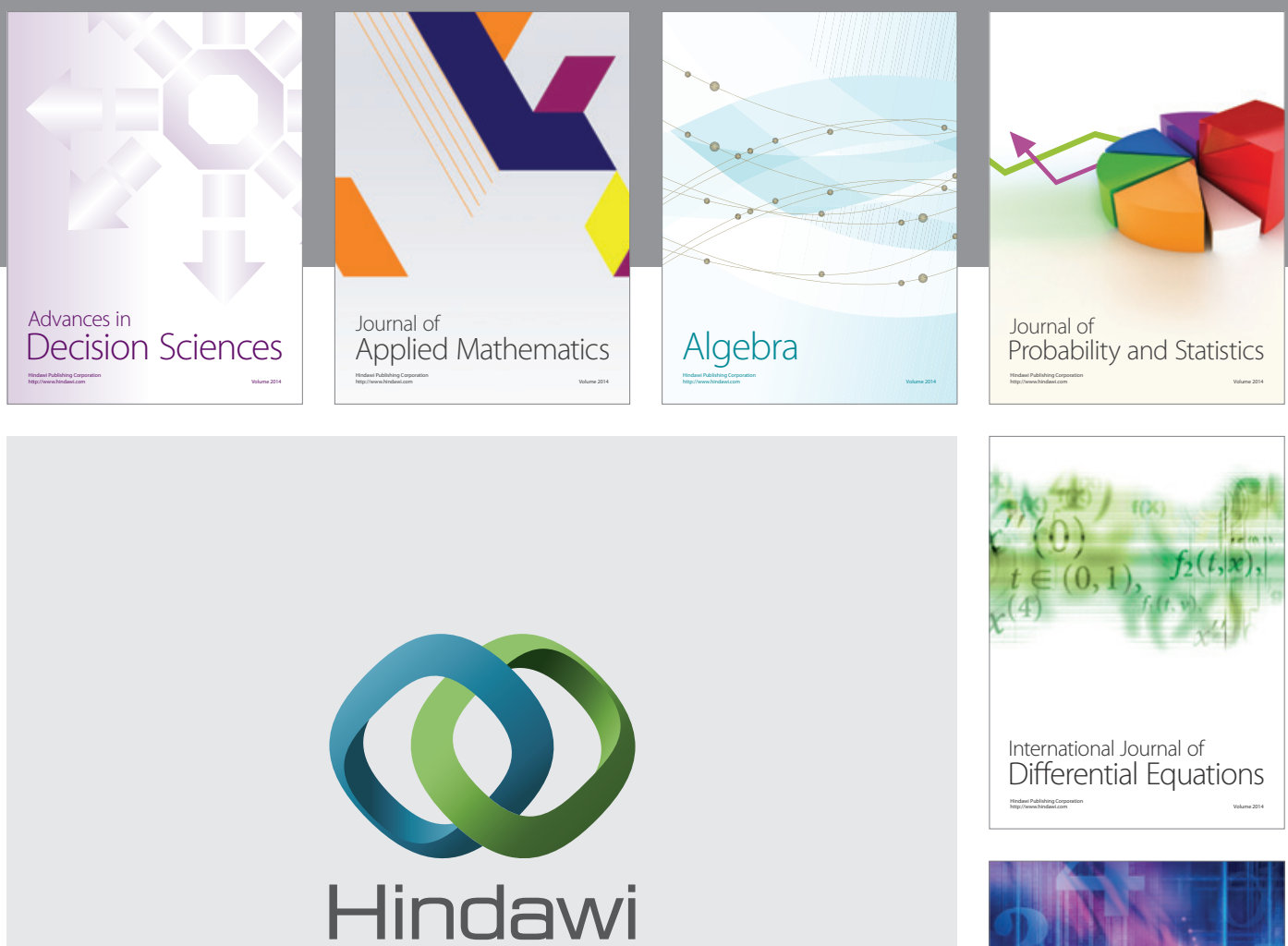

Submit your manuscripts at http://www.hindawi.com
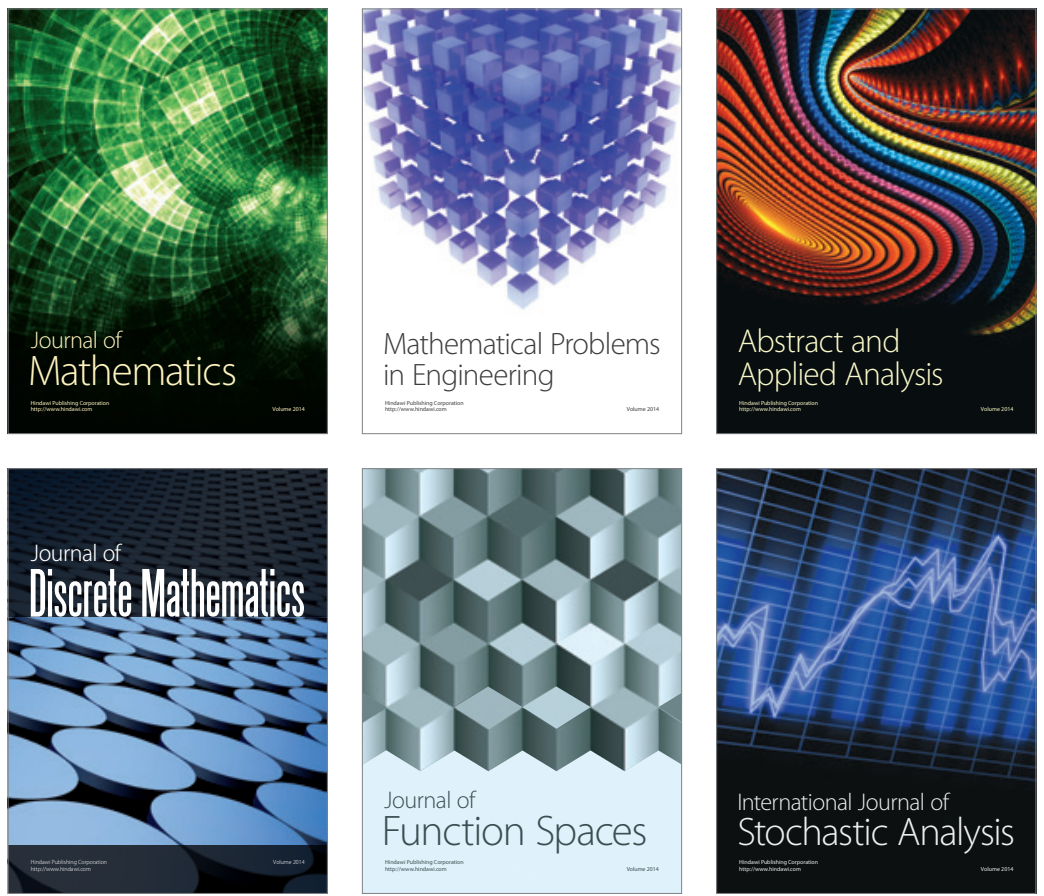

Journal of

Function Spaces

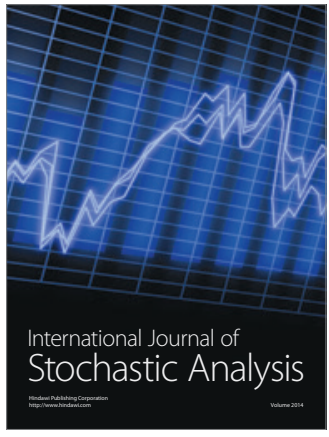

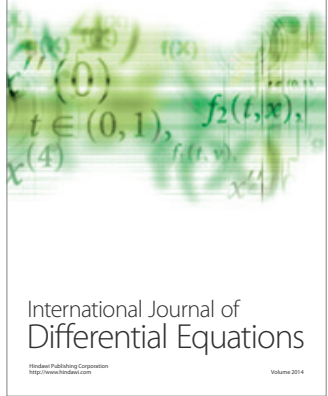
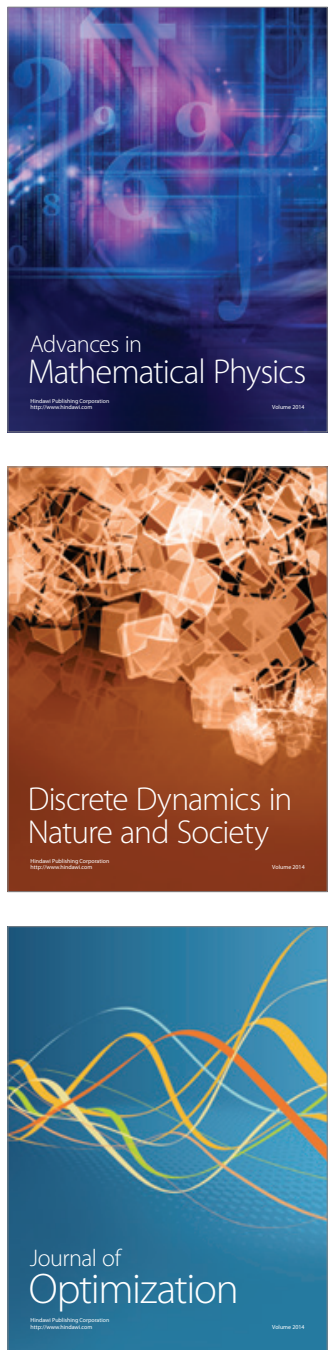